\title{
Galectin-3 in Predicting Mortality of Heart Failure: A Systematic Review and Meta-Analysis
}

\author{
Chenxia Wu, ${ }^{1,2}$ Zhengtian Lv, ${ }^{1,2}$ Xinyi Li, ${ }^{1,2}$ Xinbing Zhou, ${ }^{1,2}$ Wei Mao, ${ }^{1,2}$ Min Zhu ${ }^{1,2}$ \\ ${ }^{1}$ Department of Cardiology, The First Affiliated Hospital of Zhejiang Chinese Medical University, Hangzhou, China: ${ }^{2}$ Key \\ Laboratory of Integrative Chinese and Western Medicine for the Diagnosis and Treatment of Circulatory Diseases of Zhejiang \\ Province, Hanzhou, China
}

\section{ABSTRACT}

Background: In recent years, galectin-3, an inflammatory marker, has been demonstrated to be closely related to cardiac fibrosis and heart failure. The purpose of this systematic review and meta-analysis is to define galectin-3 in predicting mortality of heart failure.

Methods: PubMed, Embase, and the Cochrane Library were searched. A total of 1540 studies were identified, and of these studies, 19 involving 9217 patients were included in our meta-analysis.

Results: The diagnostic hazard ratios of galectin-3 in predicting mortality in chronic heart failure patients was 1.13 (95\% CI,:1.07-1.21) and 2.17 (95\% CI:1.27-3.08) in acute heart failure (HF) patients.

Conclusions: Our meta-analysis shows that elevated levels of galectin-3 are associated with higher mortality in both acute and chronic heart failure patients.

\section{INTRODUCTION}

Heart failure (HF) remains a global major public health issue, despite the increasing costs of healthcare and improvement in medical technology [Benjamin 2019]. Owing to the aging and growing population, HF prevalence is inevitably increasing worldwide [The 2018]. HF is a complex cardiovascular disease with a variety of etiologies and heterogeneity. Despite advances in treatment, there remain well-recognized morbidity and mortality. Currently, physician-assessed New York Heart Association (NYHA) functional class, B-type natriuretic peptide (BNP), and left ventricular ejection fraction (LVEF) are used in clinical as an evaluation index. However, all of them do not fully predict the risk of prognosis in HF patients [Levy 2006; Diez 2017]. Looking for a more reliable risk assessment would be of great clinical value.

Galectin-3, a member of the lectins family secreted by macrophage, is a group of proteins that interact specifically

Received December 17, 2020; accepted February 18, 2021

Correspondence: Min Zhu and Wei Mao, Department of Cardiology, The First Affiliated Hospital of Zhejiang Chinese Medical University, Hangzhou, China; (email:maoweilw@163.com,zhumin72@126.com) with carbohydrate sugars beta-galactosides [Gaggin 2013]. In recent years, emerging evidence has indicated that galectin-3 plays a role in the pathophysiology of HF [Januzzi 2005]. Galectin-3 increasing in the heart is correlated with pathophysiological states [Kim 2007]. We therefore performed a meta-analysis to evaluate the performance of plasma galectin-3 in predicting mortality in patients with HF.

\section{MATERIALS AND METHODS}

Literature search: We performed this systematic review and meta-analysis in accordance with the criterion of the Preferred Reporting Items for Systematic Reviews and Meta-analysis (PRISMA) statement. We searched Embase, Pubmed, and the Cochrane Library from inception through June 23, 2019. We used the following keywords for the literature search: (galectin-3 or gal-3) and (heart failure or HF or cardiac failure or cardiac dysfunction or cardiac insufficiency). We also searched manually for any other related research. We did not have any language or date restrictions for the search.

Study selection: Two reviewers independently searched the literature and evaluated the studies for eligibility. When having any divergences, we discussed with a third reviewer. Our included studies must meet the following inclusion criteria: 1) the study design was a prospective cohort study or retrospective cohort study); 2) test of serum galectin-3 was measured; 3) using all-cause mortality as one of the endpoints and follow up for at least 6 months for chronic heart failure and acute heart failure patients; 4) the hazard ratios (HRs) and the corresponding $95 \%$ confidence intervals (CIs) are used to describe the relationship between galectin-3 and HF. If there were multiple articles deriving from the same cohort or reporting the same event, those with the largest sample and longest follow-up duration were included.

Data extraction and quality assessment: Data from included studies were combined into a predefined standardized table. The collected basic characteristic contained the first author, publication year, region, population, mean age, sex, HF etiology, LVEF and eGFR. Study quality was independently evaluated by two reviewers using NewcastleOttawa Score (NOS) checklist. We defined the quality as $\operatorname{good}(\geq 7$ stars), fair (4-6 stars), or poor ( $<4$ stars). 
Data synthesis: Studies were evaluated on the basis of similarities in baseline patient characteristics, methods, and follow-up time. $\mathrm{I}^{2}$ statistic is used to evaluate statistical heterogeneity among studies. When $\mathrm{I}^{2}>50 \%$, it was indicative of moderate heterogeneity at least. We then used a randomeffects model to perform meta-analysis. To explore the heterogeneity, we performed subgroup analysis by sample size, publication year, follow-up duration, and mean age. In order to assess publication bias, we drew a funnel plot and performed an Egger's test for each outcome. Statistical significance was defined as a 2-tailed $P$-value $\leq 0.05$. Stata version 12.0 (Stata Corporation, College Station, TX) was used for all meta-analysis.

\section{RESULTS}

Search results: A flow chart of the selection process is shown in Figure 1. (Figure 1) The initial search query identified 1540 citations, and 1269 citations were excluded, based according to title and abstract. The full text of 271 studies were retrieved for detailed review, and we finally identified 23 eligible studies to be taken in this meta-analysis.

Study characteristics and study quality: During the literature search, we found that there were some data [Felker 2012; French 2016; Clementy 2018; Cui 2018] combining all-cause mortality with cardiovascular adverse events (MACE), cardiac transplantation, or placement of a VAD. And we carefully read the original text and found that all-cause mortality accounts for a larger proportion of follow-up indicators. Thus, we combined studies with outcomes of either all-cause mortality or other indicators to perform this meta-analysis. Finally, a total of 23 [Felker 2012; French 2016; Cui 2018; Tang 2011; de Boer 2011; Lopez-Andres 2012; Gullestad 2012; Carrasco-Sanchez 2013; Anand 2013; Motiwala 2013; Lok 2013; Gruson 2014; Fiuzat 2014; Yu 2015; Nicolas 2016; Beltrami 2016; Mueller 2016; Behnes 2016; van Vark 2017; Lala 2018; Testa 2018; Zhang 2018; Binas 2018] cohort studies were involved in this meta-analysis. All studies tested plasma galectin-3 when patients were enrolled. Basic characteristics are shown in Table 1. (Table 1) Overall, our meta-analysis

Table 1. Characteristics of included studies for meta-analysis of association of galectin-3 and HF

\begin{tabular}{|c|c|c|c|c|c|c|c|c|}
\hline De Boer (2012) & Netherlands & $\mathrm{AHF}$ & $72 \pm 12$ & 65 & 38 & $34 \pm 16$ & $61 \pm 18$ & 18 months \\
\hline Felker (2012) & USA & $\mathrm{CHF}$ & 59 & 71 & 51 & $24(19-30)$ & - & 4 years \\
\hline Carrasco-Sanchez (2013) & Spain & $\mathrm{AHF}$ & $76.2 \pm 8.6$ & 39.4 & - & - & $51.0(41-77)$ & 1 year \\
\hline Anand (2013) & USA & $\mathrm{CHF}$ & $63 \pm 12$ & 76 & 55 & $26 \pm 7$ & $57 \pm 1.01$ & 1000 days \\
\hline Motiwala (2013) & USA & $\mathrm{CHF}$ & 63.3 & 84 & 50.3 & $27.3 \pm 8.5$ & $52.6 \pm 15.3$ & 1 year \\
\hline Lok (2013) & Netherlands & $\mathrm{CHF}$ & $71 \pm 0.6$ & 81.3 & 65 & - & $61 \pm 3.4$ & 8 years \\
\hline Nicolas Clementy (2015) & France & $\mathrm{CHF}$ & $63 \pm 10$ & 81 & 21 & $34 \pm 7$ & - & 1 year \\
\hline Matteo Beltrami (2016) & Italy & AHF & $77 \pm 4$ & 49 & - & $33 \pm 9$ & $45 \pm 22$ & 6 months \\
\hline Thomas Mueller (2016) & Australia & $\mathrm{AHF}$ & $76(69-82)$ & 93 & - & $40(28-48)$ & - & 1 year \\
\hline Michael Behnes (2016) & Germany & $\mathrm{CHF}$ & $67(18-96)$ & 51 & 22 & $41(30-52)$ & - & 5 years \\
\hline Benjamin French (2017) & USA & $\mathrm{CHF}$ & 57 & 66.5 & 31.1 & $30(20-43)$ & $67(50-84)$ & 4.6 years \\
\hline Laura C. vanVark (2017) & Netherlands & $\mathrm{AHF}$ & $74(65-80)$ & 63 & 49 & $30(21-41)$ & $46(34-62)$ & 325 days \\
\hline R.I. Lala (2018) & Romania & $\mathrm{AHF}$ & $64.67 \pm 11$ & 69.6 & - & - & - & 18 months \\
\hline Marzia Testa (2018) & Italy & $\mathrm{AHF}$ & $77.5 \pm 5.9$ & 75 & - & $37.8 \pm 16.6$ & $50.9 \pm 21.7$ & 18 months \\
\hline Mingliang Zhang (2018) & China & $\mathrm{AHF}$ & $57.5 \pm 15.7$ & 70.5 & 43.7 & - & $75.0(55.6-93.0)$ & 1 year \\
\hline
\end{tabular}




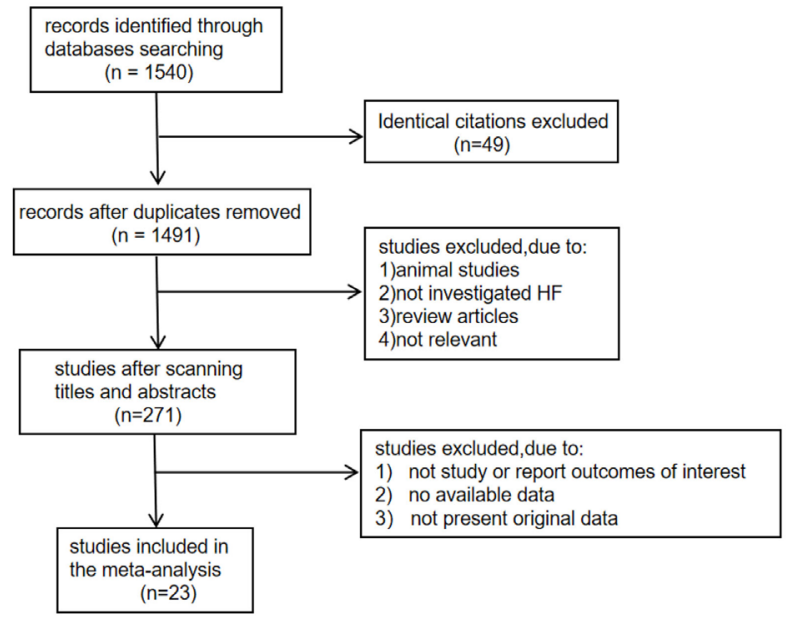

Figure 1. Flow diagram of study selection progress

included 11186 HF patients from 23 follow-up studies, and all of them were prospective cohort studies. The mean age of the patients varied from 57 to 77.5 years, and the percentage of males varied from $39 \%$ to $78.7 \%$. Follow up varied from 6 to 96 months. The meta-analysis was divided into the chronic heart failure (CHF) and acute heart failure (AHF) groups. The Newcastle-Ottawa scale varied from 7 to 9 for the included studies. (Table 2)

Statistical analysis, CHF group: Patients were eligible if they were at least 18 years of age and had heart failure for more than 3 months, with symptoms at rest or on minimal exertion (New York Heart Association functional class II or IV), and a LEVF of $40 \%$. All patients were undergoing treatment with angiotensin converting enzyme (ACE) inhibitors and digoxin for at least 3 months.

Fifteen studies [Felker 2012; French 2016; Cui 2018; Tang 2011; Lopez-Andres 2012; Gullestad 2012; Anand 2013; Motiwala 2013; Lok 2013; Gruson 2014; Fiuzat 2014; Yu 2015; Mueller 2016; Behnes 2016; Binas 2018] reported the association between serum galectin-3 and all-cause mortality. There were 8271 CHF patients along with 2448 (29.6\%) death events. Significant heterogeneity was explored $\left(\mathrm{I}^{2}=\right.$ $96.4 \%, P$ for Cochran's Q test $=0.00$ ). Pooled results with a random-effects model showed that higher serum galectin-3 on admission was independently associated with higher risk of all-cause mortality in CHF patients (HR, 1.26; 95\% CI, 1.18 to $1.36 ; P<0.001$ ) (Figure 2A)

Due to the heterogeneity among the studies, we did subgroup analysis. The results demonstrated that higher galectin-3 still was associated with increased all-cause mortality in CHF patients. (Table 3) And Egger's test demonstrated that there was no risk of publication bias in our meta-analysis $(P=0.138)$.

Statistical analysis, acute heart failure group: The AHF patients were enrolled based on the following AHF criteria: (a) dyspnea at rest or minimal effort, (b) pulmonary congestion visible on chest $\mathrm{X}$-ray, (c) ultrasound signs of interstitial edema or pleural effusion, and (d) need for $\geq 40 \mathrm{mg}$ intravenous furosemide within $16 \mathrm{~h}$ of hospital admission. a.

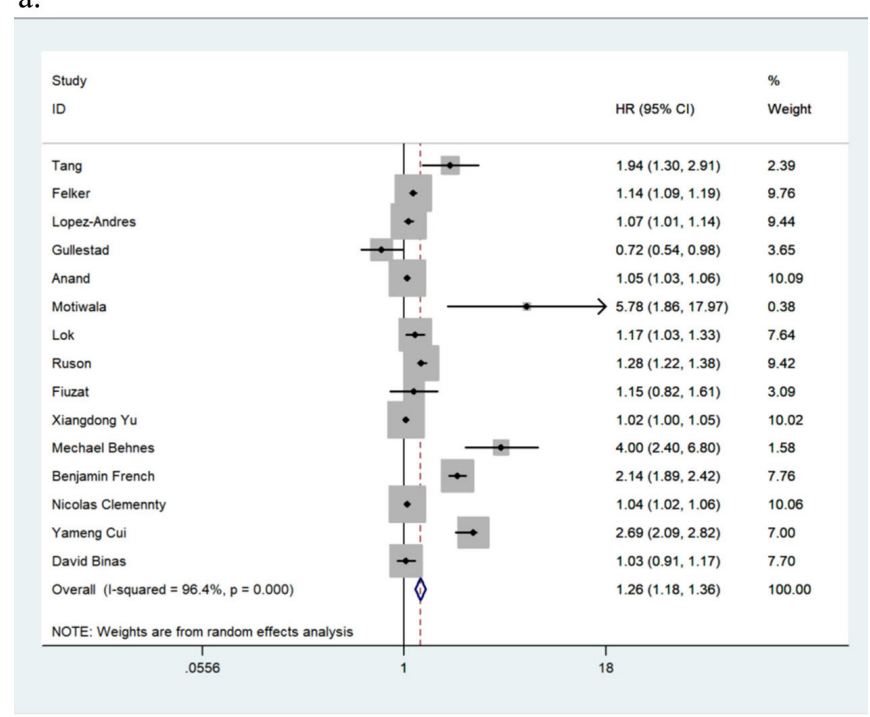

b.

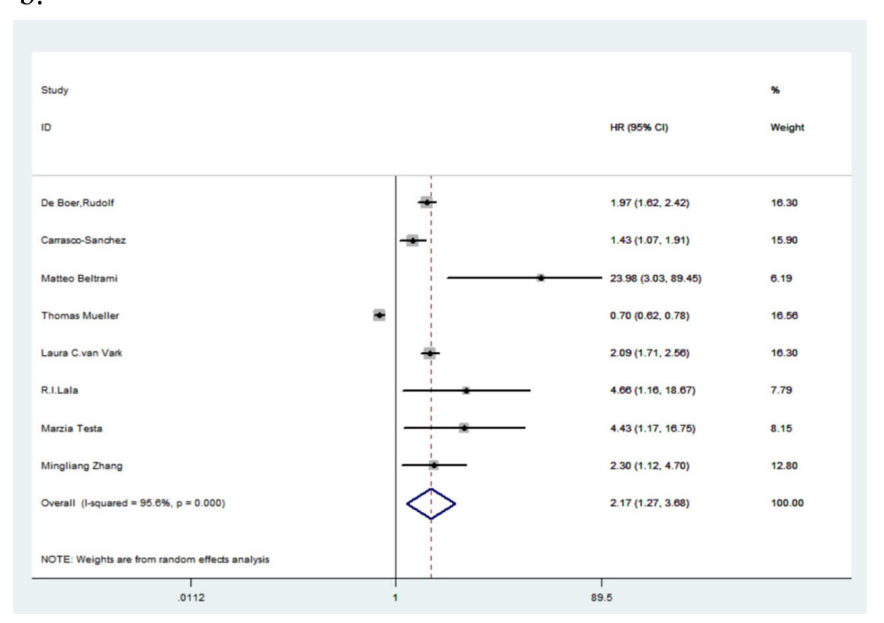

Figure 2. Forest plots for the meta-analyses of the association between serum galectin-3 and all-cause mortality in chronic HF (top) and acute HF (bottom) patients.

Eight studies [Clementy 2018; de Boer 2011; CarrascoSanchez 2013; Beltrami 2016; van Vark 2017; Lala 2018; Testa 2018; Zhang 2018] evaluated the relationship between galectin-3 and all-cause mortality of AHF, in which encountered $642(22.0 \%)$ death out of 2915 AHF patients. Using the random-effects model, the result indicated that for every $1 \%$ increase in plasma galactosin- 3 , the associated risk of allcause mortality increased by $117 \%$ (HR $2.17,95 \%$ CI 1.27 $3.08, \mathrm{I}^{2}=90.7 \%$ ). (Figure 2B)

We also performed a subgroup analysis to explore the heterogeneity among AHF studies. When grouped by publication year, follow-up duration and mean age, some showed galectin-3 was not associated with increased all-cause mortality (Table 3B). However, Egger's test demonstrated that there was no risk of publication bias in our meta-analysis for acute HF all-cause mortality $(P=0.536)$. 
Table 2. NOS risk of bias scale for included cohort studies

\begin{tabular}{|c|c|c|c|c|c|c|c|c|c|}
\hline \multirow{2}{*}{ Author (year) } & \multicolumn{4}{|c|}{ Selection } & \multirow{2}{*}{$\begin{array}{l}\text { Compar } \\
\text { ability }\end{array}$} & \multirow{2}{*}{$\begin{array}{l}\text { Assessment } \\
\text { of outcome }\end{array}$} & \multirow{2}{*}{$\begin{array}{l}\text { Adequacy of duratiom } \\
\text { of follow-up }\end{array}$} & \multirow{2}{*}{$\begin{array}{c}\text { Adequacy of } \\
\text { completeness of follow- } \\
\text { up }\end{array}$} & \multirow{2}{*}{$\begin{array}{c}\text { Total score } \\
(0-9)\end{array}$} \\
\hline & 1 & 2 & 3 & 4 & & & & & \\
\hline Tang(2011) & * & * & $*$ & $\therefore$ & $*$ & $\stackrel{n}{*}$ & $\stackrel{2}{*}$ & 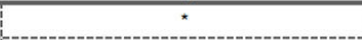 & 9 \\
\hline De Boer(2011) & $*$ & * & * & $*$ & "- & $-\bar{*}$ & $-\bar{*}$ & & 8 \\
\hline Felker(2012) & * & * & $*$ & $*$ & $*$ & 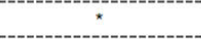 & 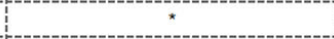 & * & 9 \\
\hline Lopez-Andrès(2012) & $*$ & $*$ & $*$ & $*$ & $*$ & $\cdots$ & $\therefore$ & 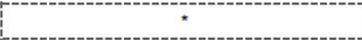 & 9 \\
\hline Gullestad(2012) & * & $*$ & $*$ & $\cdots$ & 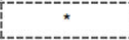 & $\stackrel{-*}{*}$ & 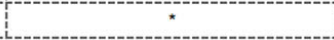 & 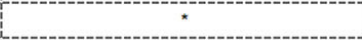 & 9 \\
\hline Carrasco-Sánchez $(2013)$ & $\times$ & 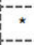 & 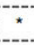 & 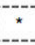 & 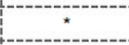 & $-\cdots$ & & & 7 \\
\hline Anand (2013) & * & $*$ & $*$ & $*$ & $*$ & * & * & * & 9 \\
\hline Motiwala(2013) & * & * & $*$ & 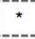 & 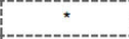 & 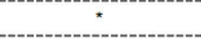 & & $*$ & 8 \\
\hline Lok(2013) & $*$ & : & $*$ & $*$ & $*$ & $\therefore$ & 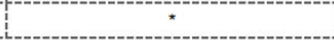 & $*$ & 9 \\
\hline Ruson(2014) & $*$ & $*$ & $*$ & $\cdots$ & $*$ & * & $\cdots$ & * & 9 \\
\hline Fiuzat(2014) & * & * & $*$ & $\cdots$ & $*$ & 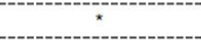 & 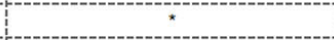 & 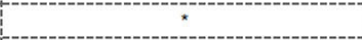 & 9 \\
\hline Xiangdong Yu(2015) & $*$ & * & $*$ & $*$ & $*$ & $\therefore$ & - & 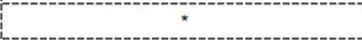 & 8 \\
\hline Nicolas Clementy (2015) & 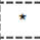 & : & $*$ & $\cdots$ & $*$ & $\therefore$ & $\cdots$ & $*$ & 9 \\
\hline Matteo Beltrami (2016) & $-*$ & : & $*$ & 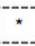 & 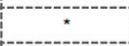 & 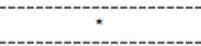 & & 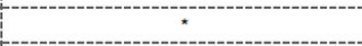 & 8 \\
\hline Thomas Mueller(2016) & $*$ & * & $*$ & $*$ & $*$ & 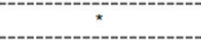 & & $-\bar{*}$ & 8 \\
\hline Michael Behnes (2016) & $*$ & : & $*$ & $*$ & 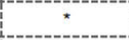 & $\therefore$ & 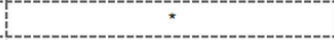 & 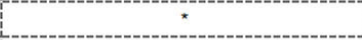 & 9 \\
\hline Benjamin French (2017) & * & * & $*$ & $*$ & $*$ & 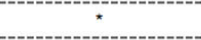 & * & $*$ & 9 \\
\hline Laura C. van Vark(2017) & $-*$ & : & $*$ & $\cdots$ & 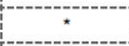 & $\cdots$ & & 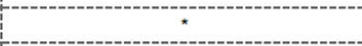 & 8 \\
\hline R. I. Lala(2018) & 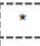 & : & * & 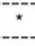 & $-\bar{x}^{-}$ & $-\bar{*}$ & $-\bar{*}$ & $-\bar{x}$ & 9 \\
\hline Marzia Testa (2018) & $*$ & : & 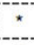 & 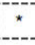 & 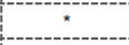 & --" & $-{ }^{*}$ & & 8 \\
\hline Mingliang Zhang (2018) & 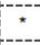 & : & $\cdots$ & $\cdots$ & $\therefore$ & $\therefore$ & & $\therefore$ & 8 \\
\hline Yameng Cui (2018) & * & * & $*$ & $\cdots$ & $\therefore$ & 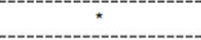 & 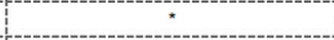 & 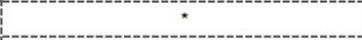 & 9 \\
\hline David Binas (2018) & $*$ & $\star *$ & * & $*$ & * & * & * & * & 9 \\
\hline
\end{tabular}

Attention: 1.representativeness of exposed cohort; 2.selection of non-exposed cohort; 3.limitation of exposure;4.no ending events occurred at the start of the study

\section{DISCUSSION}

The findings from our data are that in HF: (a) Galectin-3 is associated with prognosis in patients with HF; (b) Galectin-3 has a greater impact on the prognosis of AHF patients.

Fibroblasts and macrophages are responsible for the initiation and progression of tissue fibrosis [Gaggin 2013]. It is linked to disease progression and poor prognosis. Cardiac fibrosis is an important contributor to the pathophysiology of cardiac remodeling. Cardiac remodeling is an essential feature of $\mathrm{HF}$ and is closely linked to disease progression [Brown 2005]. Increases in galectin-3 levels in organs are correlated with pathophysiological states. And galectin-3 secreted by macrophages and fibroblasts participate in the maladaptive pathological cardiac responses, such as remodeling, fibroblast proliferation, and collagen deposition [Sharma 2004]. Recent research demonstrated that inhibition of galectin 3 improves cardiomyocyte apoptosis and survival during heart failure [ $\mathrm{Li} 2018]$. The potential utility of galectin-3 in HF prognosis has been attained, and galectin-3 likely represents a unique phenotype at high risk for the development and progression of heart failure.

In order to highlight the possible utility of plasma galectin-3 as a biomarker, De Boer et al. demonstrated that galectin-3 to be a novel biomarker of adverse outcomes (all-cause mortality and HF hospitalization) in HF patients (HR 1.38, 95\% CI $1.07-1.78, P=0.015$ ) [de Boer 2011]. And Nguyen et al. found that the expression of galectin-3 and the density of inflammatory cells in the heart were significantly elevated in heart disease mouse model compared with the wild-type mice [Jabagi 2019]. Chen et al. and Imran et al. conducted meta-analysis on galectin-3's ability as a prognostic marker for CHF and AHF, concluded that increased galectin-3 is associated with higher longterm all-cause mortality HF [Chen 2016; Imran 2017]. In recent years, there is a rising interest in the discovery to fulfill this role, so we want to make an update and further meta-analysis.

As we all know, renal dysfunction is closely related to HF. Galectin-3 expressed in kidneys and heart is correlated with pathophysiological states. Although several prognostic studies of patients with $\mathrm{HF}$ have indicated the independent predictive value of galectin 3 for cardiovascular outcomes, others have questioned whether galectin 3 is truly independently associated with adverse cardiac events [Anand 2013; de Boer 2009]. In our data, half of included studies recorded the eGRF, and most of them were less than moderate renal dysfunction. Therefore, we believe that renal function did not have a great impact on the results of our meta-analysis, but still cannot use plasma galectin-3 as an independent factor to judge the prognosis of $\mathrm{HF}$.

\section{LIMITATIONS}

Some limitations must be noted. First, this review has high heterogeneity. We performed subgroup analysis by different factors, and it had statistically significant results. Second, the short follow-up time in some studies included in the all-cause mortality analysis may have influenced these results. 
Table 3. Subgroup analysis for the hazard ratio meta-analyses of galectin-3 and all-cause mortality in chronic HF (A) and acute HF (B)

\begin{tabular}{|c|c|c|c|}
\hline \multicolumn{4}{|l|}{ A } \\
\hline$\leq 400$ & 9 & $1.26(1.14-1.40)$ & $96.3 \% P<0.001$ \\
\hline$>400$ & 6 & $1.32(1.10-1.58)$ & $97.0 \% P<0.001$ \\
\hline$\leq 2013$ & 7 & $1.10(1.02-1.19)$ & $84.3 \% P<0.001$ \\
\hline$>2013$ & 8 & $1.44(1.26-1.65)$ & $98.0 \% P<0.001$ \\
\hline \multicolumn{4}{|c|}{ Follow-up duration } \\
\hline$\leq 3$ years & 8 & $1.16(1.07-1.24)$ & $95.9 \% P<0.001$ \\
\hline$>3$ years & 7 & $1.46(1.22-1.76)$ & $95.1 \% P<0.001$ \\
\hline \multicolumn{4}{|l|}{ B } \\
\hline \multicolumn{4}{|l|}{ Sample size } \\
\hline$\leq 400$ & 4 & $3.84(0.71-20.71)$ & $90.2 \% P<0.001$ \\
\hline$>400$ & 4 & $1.88(1.58-2.24)$ & $39.4 \% P=0.176$ \\
\hline \multicolumn{4}{|c|}{ Publication year } \\
\hline$\leq 2016$ & 4 & $1.75(0.85-3.63)$ & $97 \% P<0.001$ \\
\hline$>2016$ & 4 & $2.17(1.79-2.62)$ & $0 \% P=0.489$ \\
\hline \multicolumn{4}{|c|}{ Follow-up duration } \\
\hline
\end{tabular}

\section{ACKNOWLEDGMENTS}

This work was supported by the National Natural Science Foundation of China (Grant no.81973579).

\section{CONCLUSION}

Our results show that acute or chronic heart failure patients with high serum level of galectin-3 gain more mortality.

\section{REFERENCES}

Anand IS, et al. 2013. Baseline and serial measurements of galectin-3 in patients with heart failure: relationship to prognosis and effect of treatment with valsartan in the Val-HeFT. Eur J Heart Fail. 15(5): p. 511-8.

Behnes M, et al. 2016. Triple head-to-head comparison of fibrotic biomarkers galectin-3, osteopontin and gremlin-1 for long-term prognosis in suspected and proven acute heart failure patients. Int J Cardiol. 203: p. 398-406.

Beltrami M, et al. 2016. Additional value of Galectin-3 to BNP in acute heart failure patients with preserved ejection fraction. Clin Chim Acta. 457: p. 99-105.

Benjamin EJ, et al. 2019. Heart Disease and Stroke Statistics-2019 Update: A Report From the American Heart Association. Circulation. 139(10): p. e56-e528.

Binas D, et al. 2018. The prognostic value of sST2 and galectin-3 considering different aetiologies in non-ischaemic heart failure. Open Heart. 5(1): p. e000750.

Brown RD, et al. 2005. The cardiac fibroblast: therapeutic target in myocardial remodeling and failure. Annu Rev Pharmacol Toxicol. 45: p. 657-87.

Carrasco-Sanchez FJ, et al. 2013. Predictive value of serum galectin-3 levels in patients with acute heart failure with preserved ejection fraction. Int J Cardiol. 169(3): p. 177-82. 
Chen YS, et al. 2016. Using the galectin-3 test to predict mortality in heart failure patients: a systematic review and meta-analysis. Biomark Med. 10(3): p. 329-42.

Clementy N, et al. 2018. Galectin-3 level predicts response to ablation and outcomes in patients with persistent atrial fibrillation and systolic heart failure. PLoS One. 13(8): p. e0201517.

Cui Y, et al. 2018. Differential and Predictive Value of Galectin-3 and Soluble Suppression of Tumorigenicity-2 (sST2) in Heart Failure with Preserved Ejection Fraction. Med Sci Monit. 24: p. 5139-5146.

de Boer RA, et al. 2009. Galectin-3: a novel mediator of heart failure development and progression. Eur J Heart Fail. 11(9): p. 811-7.

de Boer RA, et al. 2011. Predictive value of plasma galectin-3 levels in heart failure with reduced and preserved ejection fraction. Ann Med. 43(1): p. $60-8$.

Diez J. 2017. Chronic heart failure as a state of reduced effectiveness of the natriuretic peptide system: implications for therapy. Eur J Heart Fail. 19(2): p. 167-176.

Felker GM, et al. 2012. Galectin-3 in ambulatory patients with heart failure: results from the HF-ACTION study. Circ Heart Fail. 5(1): p. 72-8.

Fiuzat M, et al. 2014. Relationship between galectin-3 levels and mineralocorticoid receptor antagonist use in heart failure: analysis from HFACTION. J Card Fail. 20(1): p. 38-44.

French B, et al. 2016. Prognostic Value of Galectin-3 for Adverse Outcomes in Chronic Heart Failure. J Card Fail. 22(4): p. 256-62.

Gaggin HK and Januzzi JL, Jr. 2013. Biomarkers and diagnostics in heart failure. Biochim Biophys Acta. 1832(12): p. 2442-50.

Gruson D, et al. 2014. Measurement of Galectin-3 with the ARCHITECT assay: Clinical validity and cost-effectiveness in patients with heart failure. Clin Biochem. 47(12): p. 1006-9.

Gullestad L, et al. 2012. Galectin-3 predicts response to statin therapy in the Controlled Rosuvastatin Multinational Trial in Heart Failure (CORONA). Eur Heart J. 33(18): p. 2290-6.

Imran TF, et al. 2017. Meta-Analysis of the Usefulness of Plasma Galectin-3 to Predict the Risk of Mortality in Patients With Heart Failure and in the General Population. Am J Cardiol. 119(1): p. 57-64.

Jabagi H, et al. 2019. Biomarkers in the Diagnosis, Management, and Prognostication of Perioperative Right Ventricular Failure in Cardiac Surgery-Are We There Yet? J Clin Med. 8(4).

Januzzi JL, Jr. et al. 2005. The N-terminal Pro-BNP investigation of dyspnea in the emergency department (PRIDE) study. Am J Cardiol. 95(8): p. $948-54$.

Kim H, et al. 2007. Expression and immunohistochemical localization of galectin-3 in various mouse tissues. Cell Biol Int. 31(7): p. 655-62.
Lala RI, et al. 2018. Galectin-3 as a marker for clinical prognosis and cardiac remodeling in acute heart failure. Herz. 43(2): p. 146-155.

Levy WC, et al. 2006. The Seattle Heart Failure Model: prediction of survival in heart failure. Circulation. 113(11): p. 1424-33.

Li X, et al. 2018. Therapeutic inhibition of galectin3 improves cardiomyocyte apoptosis and survival during heart failure. Mol Med Rep. 17(3): p. 4106-4112.

Lok DJ, et al. 2013. Galectin-3 is an independent marker for ventricular remodeling and mortality in patients with chronic heart failure. Clin Res Cardiol. 102(2): p. 103-10.

Lopez-Andres N, et al. 2012. Association of galectin-3 and fibrosis markers with long-term cardiovascular outcomes in patients with heart failure, left ventricular dysfunction, and dyssynchrony: insights from the CAREHF (Cardiac Resynchronization in Heart Failure) trial. Eur J Heart Fail. 14(1): p. 74-81.

Motiwala SR, et al. 2013. Serial measurement of galectin-3 in patients with chronic heart failure: results from the ProBNP Outpatient Tailored Chronic Heart Failure Therapy (PROTECT) study. Eur J Heart Fail. 15(10): p. 1157-63.

Mueller T, et al. 2016. Diagnostic and prognostic accuracy of galectin-3 and soluble ST2 for acute heart failure. Clin Chim Acta. 463: p. 158-164.

Nicolas D, et al. 2016. [Respiratory failure caused by amyloid myopathy]. Med Clin (Barc). 146(5): p. e25-6.

Sharma UC, et al. 2004. Galectin-3 marks activated macrophages in failure-prone hypertrophied hearts and contributes to cardiac dysfunction. Circulation. 110(19): p. 3121-8.

Tang WH, et al. 2011. Usefulness of plasma galectin-3 levels in systolic heart failure to predict renal insufficiency and survival. Am J Cardiol. 108(3): p. 385-90.13.

Testa M, et al. 2018. The Predictive Value of Plasma Brain Natriuretic Peptide and Galectin-3 in Elderly Patients Admitted for Heart Failure. Diseases. 6(4).

The L. 2018. Heart failure: the need for improved treatment and care. Lancet. 392(10146): p. 451.

van Vark LC, et al. 2017. Prognostic Value of Serial Galectin-3 Measurements in Patients With Acute Heart Failure. J Am Heart Assoc. 6(12).

Yu X, et al. 2015. Prognostic value of plasma galectin-3 levels in patients with coronary heart disease and chronic heart failure. Int Heart J. 56(3): p. 314-8.

Zhang M, et al. 2018. Comparison of multiple biomarkers for mortality prediction in patients with acute heart failure of ischemic and nonischemic etiology. Biomark Med. 12(11): p. 1207-1217. 